\title{
A RBF Network for Chinese Text Classification Based on Concept Feature Extraction
}

\author{
Minghu Jiang ${ }^{1,2}$, Lin Wang ${ }^{1,3}$, Yinghua $\mathrm{Lu}^{1}$, and Shasha $\mathrm{Liao}^{2}$ \\ ${ }^{1}$ School of Electronic Eng., Beijing University of Post and Telecommunication, \\ Beijing, 100876, China \\ ${ }^{2}$ Lab. of Computational Linguistics, School of Humanities and Social Sciences, \\ Tsinghua University, Beijing 100084, China \\ ${ }^{3}$ Interdisciplinary Center for Scientific Computing (IWR), University of Heidelberg, \\ Im Neuenheimer Feld 368, 69210 Heidelberg, Germany \\ jiang.mh@t.singhua.edu.cn
}

\begin{abstract}
The feature selection is an important part in automatic text classification. In this paper, we use a Chinese semantic dictionary -- Hownet to extract the concepts from the word as the feature set, because it can better reflect the meaning of the text. We construct a combined feature set that consists of both sememes and the Chinese words, propose a CHI-MCOR weighing method according to the weighing theories and classification precision. The effectiveness of the competitive network and the Radial Basis Function (RBF) network in text classification are examined. Experimental result shows that if the words are extracted properly, not only the feature dimension is smaller but also the classification precision is higher, the RBF network outperform competitive network for automatic text classification because of the application of supervised learning. Besides its much shorter training time than the BP network's, the RBF network makes precision and recall rates that are almost at the same level as the BP network's.
\end{abstract}

Keywords: text classification, concept feature, RBF network.

\section{Introduction}

Automatic text classification is a process which classifies the documents to several categories by their content. With the rapid development of the online information, automatic text classification becomes one of the key techniques for handling and organizing text data. It always includes two main parts: the feature selection and the classifier. Because of the huge data of the document sets, it is difficulty for reflecting the feature vector of documents, we need to construct a proper feature set which is easily process and has a considerable classification precision. Because the concept space is much smaller than the word one, and the components are comparatively independent, the concept features are much better to reflect the content of the documents. With the semantic analysis we can get a much better vector space [1], therefore we choose semantic features as the main components of our feature set.

Many techniques can be used in feature selection to improve accuracy as well as reduce the dimension of the feature vector and thus reduce the time of computation

I. King et al. (Eds.): ICONIP 2006, Part III, LNCS 4234, pp. 285 - 294, 2006.

(c) Springer-Verlag Berlin Heidelberg 2006 
$[2,3]$. These techniques include using concept frequency instead of original word frequency and Latent Semantic Indexing (LSI) [3]. However, after using these techniques, the feature matrix is still quite complicated and requires a robust classifier to deal with.

Many classifiers have been applied to classify texts, including Vector Space Models, K-Means, Support Vector Machine (SVM), Naïve Bayes and so on [3]. Among many methods applied, several kinds of neural networks have shown attractive abilities. The competitive networks are used in text classification, including Learning Vector Quantization (LVQ) and Self-Organizing Maps (SOM) network [4, 5]. These two are variants of the unsupervised competitive networks. Besides, the Radial Basis Function (RBF) network, which is characterized by its high speed of training, can also be used in text classification.

In this paper we make a performance comparison between the competitive network, and RBF network in text classification. For unsupervised competitive network, we use the evaluation criterion, and define positive and negative accuracies and use their average to evaluate the performance of clustering.

Table 1. Definition of positive and negative accuracy

\begin{tabular}{lll}
\hline & Clustered together & Not clustered together \\
\hline In same category & $A$ & $\mathrm{C}$ \\
In different category & $B$ & $D$ \\
\hline
\end{tabular}

Positive Accuracy $A_{p}=A /(A+C)$, and negative accuracy $A_{n}=D /(B+D)$. Average Accuracy $A_{a}=\left(A_{p}+A_{n}\right) / 2$. For the supervised classification networks, the factors we take into account are time, precision, recall and $F 1$. Precision and recall are two widely used criteria of evaluation in text classification and text retrieval. Let us assume that $P$ implies precision, $R$ implies recall, $A_{i}$ is the number of documents in category $i$, and $B_{i}$ is the number of the documents classified by the classifier to category $i$, then $p_{i}=\frac{A_{i} \cap B_{i}}{B_{i}}$, and $R_{i}=\frac{A_{i} \cap B_{i}}{A_{i}} . F 1$ is a criterion that combines precision and recall, $F 1=\frac{2 p \times R}{P+R}$.

\section{Concept Feature Extraction}

Hownet is an on-line common-sense knowledge base which unveil the interconceptual relations and the inter-attribute relations of concepts as connoting in lexicons of the Chinese and their English equibalents [6]. Different from Wordnet, the concept definitions in Hownet are not in a tree form in which the distance between them can be directly calculated, instead, they are in multidimensional forms. The concept definition is a description of the word semantic, and one word may have several concept definitions, reflecting several different meanings. The definitions consist of a series of sememes, which refer to the smallest basic semantic unit, and these sememes are divided to nine main categories, such as Entity and Event categories, and they have Hypernym-Hyponym relations among them. 


\subsection{Tree Form of Sememes and Expression Power of Sememes}

As far as the sememes in Hownet have Hypernym-Hyponym relations, the father node is always abstract than the child node. The accessional tables in Hownet give the Hypernym-Hyponym relations of the 1505 sememes, and we can build several sememe trees for further process. In these trees, every node refers to one sememe, and its position shows its relation to other sememes. We take three factors into consideration for the expression power. First, the nodes in different level should have different expression power, the different value should be given when we extract them from the word. Second, if a sememe has more child nodes, its value should be more reduced, as it can be divided into several detailed concepts in Hownet, and its child nodes would be chosen to describe the word unless the concept is abstract. Third, the different sememe trees should have different expression powers, the different tree roots have the different original values. For example, the Attribute tree may has small expression powers, while Entity tree are strong in description and their root value is higher. The formula is shown as follows:

$$
k(m)=\text { Wtree }_{i} \cdot\left[\log \left(\left(\text { Deep }_{k}+1.0\right) / 2\right)+\mathrm{a}+\frac{1}{\text { Child }_{k}+\mathrm{b}}\right] .
$$

Where, $k(m)$ is the expression power of sememe $m ; k$ is the node referring to $m$ in the sememe tree; Wtree $_{i}$ is the root value of tree $i$ which the node $k$ is in; Deep is $_{k}$ the height of the node $k$. Because the difference among different levels would be too much if the height is used as their weighing value, therefore we use the logarithm value of the height. Meanwhile, as the range of the tree height is from 0 to 12 , we divided the value by 2 in order to make the result better; Child ${ }_{k}$ is the number of its child nodes, its range is from 0 to 9 , and we set a mediating factor, $b$, to protect the calculation from invalid value; $a$ is also a mediating factor, which avoid the result being too small. The reflection between the sememe $m$ and the node $k$ can be found in the corresponding table in our system, and the height and child node number are also described in an index table.

\subsection{Expression Power of Concept Definition}

As there are some sememes in Hownet which are abstract and occur in a lot of definitions of the words, and do not contain enough information to describe them, we need to separate the sememes into two kinds according to their expression power, the strong one and the weak one. If the definition of a certain word contains only weak ones, it means that it does not describe the word accurately and the information gain is not enough, thus we should not extract the words to their definition. To calculate the expression power of a definition, we set a threshold to filter the weak ones and reserve the word which cannot be extracted. In this way, the feature set consists of both words and sememes, the expression power of definition $c$ can be calculated as follows:

$$
\mathrm{f}(c)=\max _{j} k\left(c_{j}\right)
$$

Where $k\left(c_{j}\right)$ is the weight of sememe $j$ in concept definition $c$; (2) calculates the expression power of all the sememes, and chooses the highest value as the expression power of the definition. 


\subsection{Threshold in Concept Extraction}

To every definition, if its expression power is high enough, we can extract it from the word, otherwise, it should not be extracted. In order to decide when to extract it, we need a threshold to divide the definition into two parts, which can be extracted or cannot. When the definite value is higher than the threshold, this word is extracted and the sememes are added into the feature set. Otherwise, the original word is added instead.

\subsection{Combined Weighing Method: CHI-MCOR}

Analysis of feature set. When we extract the concept attribute to construct the feature set, we convert a lot of words into the concept features, and get rid of the influence of the synonymy and dependence, which makes the classification precision much higher. However, because of the mass of weak concept and the words which are not in the Hownet, some Chinese words are given a comparatively lower weight and become the middle or low occurring feature. There are still some specialty words and proprietary words which are only occur in one category and are not highly occurred in the whole documents, however these words are very important in classification. These words need a strategy to get a higher weight and contribute more in text classification, we propose the weighing methods.

The comparing result of seven weighing methods. We select seven common weighing methods and test them, and focus mainly on their selection strategy and classification precision. The table 1 gives us the experimental results. From the analysis of the selected features, we find the following facts.

Fact 1. The DF (Document Frequency), TF-IDF (Term Frequency-Inverse Document Frequency), CET (an improved method of Information Gain), CDW (CategoryDiscriminating Word) and CHI ( $\chi$ statistics) methods prefer the high-occurred words and they are greatly related. In our experiment, $\mathrm{CHI}$ is the best method.

Fact 2. The MCOR (Multi-Class Odds Ratio) method mainly chooses the middle and low occurred features, so its classification precision is low when the reduction rate is high. But with the increase of the feature dimension, its precision is increased highly and when the feature dimension is above 4000, its precision is higher than CDW, CET, DF, TF-IDF and MI (Mutual Information) methods.

Fact 3. The MI method mainly selects the high and middle occurred features, it can get a good classification precision but with the increase of the feature dimension, the precision is not improved visibly.

The CHI selection method. The CHI weighing method's formula is shown as follow:

$$
\begin{gathered}
\chi^{2}(t, c)=\frac{N *(A D-C B)^{2}}{(A+C)(B+D)(A+B)(C+D)} . \\
\mathrm{x}_{\max }^{2}(t)=\max _{i=1}^{m} \chi^{2}\left(t, c_{i}\right) .
\end{gathered}
$$




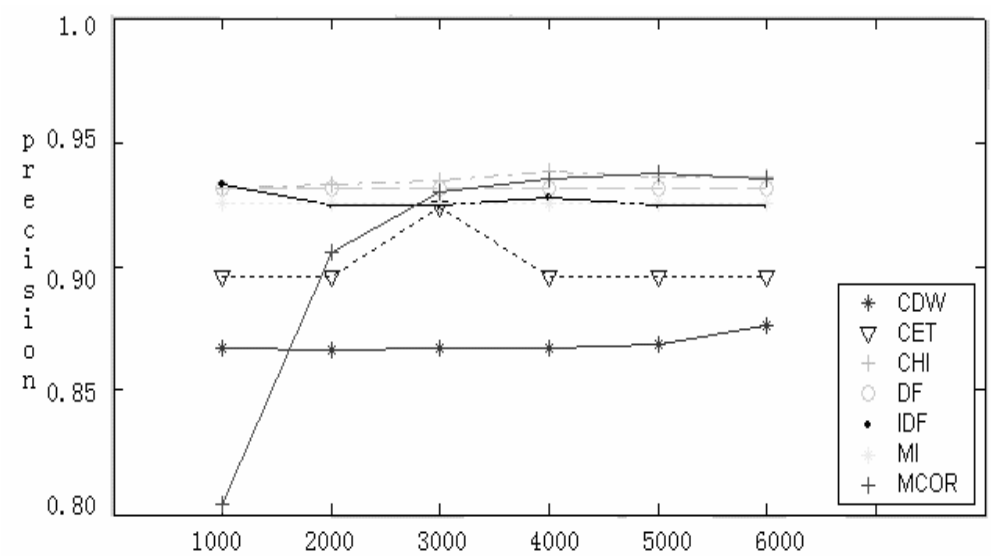

Fig. 1. The average of seven different weighing methods. $\mathrm{Y}$ axis is the average precision, and $\mathrm{x}$ axis is the feature dimension of the training set.

Where, $N$ is the total document number of the training set, $c$ is a certain category, $t$ is a certain feature, $A$ is the number of the document which belong to category $c$ and contain feature $t, B$ is those which do not belong to category $c$ but contain feature $t, C$ is those which belong to category $c$ but do not contain feature $t, D$ is those which do not belong to category $c$ and do not contain feature $t$.

The CHI method is based on such hypothesis: if the feature is highly occurred in a specified category or highly occurred in other categories, it is useful for classification. Because CHI take the occurrence frequency into account, it prefers to select highly occurred words, and ignored the middle and low occurred words which maybe important in classification.

The MCOR selection method. The MCOR weighing method's formula is shown as follow [1]:

$$
\operatorname{MCOR}(t)=\sum_{i=1}^{m} P\left(C_{i}\right)\left|\log \frac{P\left(t / C_{i}\right)\left(1-P\left(t / C_{\text {else }}\right)\right.}{\left(1-P\left(t / C_{i}\right) P\left(t / C_{\text {else }}\right)\right.}\right|
$$

Where, $P\left(C_{i}\right)$ is the occurrence probability of category $C_{i}, P\left(t / C_{i}\right)$ is the occurrence probability of the feature $t$ when category $C$ is occurred, $P\left(t / C_{\text {else }}\right)$ is the occurrence probability of the feature $t$ when category $C$ is not occurred. When $P\left(t / C_{i}\right)$ is higher or $P\left(t / C_{\text {else }}\right)$ is lower, the weight of MCOR is higher. Therefore, the MCOR selects the features which are mainly occurred in one category and nearly not occurred in other categories. Because it does not consider the occurrence frequency of the feature, it prefer to select the words which are middle or low occurred in the document while highly occurred words are always occurred in more than one categories.

The Combined Method: CHI-MCOR. Because MCOR mainly selects the words whose occurrence frequencies are middle or low, its classification precision is low when the reduction is high. But with the increase of feature dimension, its precision is improved to an appreciable level. And CHI prefers to select the words whose 
occurrence frequencies are high, and it is one of the best feature selection methods [1]. As a result, when we combine the both methods, we can make the advantages together and get a high classification precision [7]. Therefore, we give a combined weighing method based on CHI and MCOR, it is shown as follows [8]:

$$
V(t)=\lambda V_{C H I}(t)+(1-\lambda) V_{M C O R}(t), \quad 0<\lambda<1 .
$$

Where, $V_{C H I}$ is the weight of feature $t$ when CHI method is used, $V_{M C O R}$ is that when MCOR method is used.

When we analysis the weigh given by the both methods, we find that the average weight of the features are different. For example, when the reduction is $50 \%$, the range of the CHI weight is $(2.1,6.81)$, while that of MCOR is $(1.15,1.76)$. Because $\mathrm{CHI}$ gives a much higher weight to all the features and its swing is wider, we should give a comparatively lower value to $\lambda$. If not, the value depends too much on $\mathrm{CHI}$ and the combined weigh method is meaningless. So we need a proper value of $\lambda$. In experience, we suppose that when the average weight of CHI and MCOR is the same, we can get the both advantage and the classification precision will be the highest. Therefore, we think that the best $\lambda$ is as follows:

$$
\frac{\lambda}{1-\lambda}=\frac{\operatorname{Mean}(\mathrm{MCOR})}{\operatorname{Mean}(\mathrm{CHI})} \text {. }
$$

\subsection{Competitive Learning Network}

Competitive learning network usually consists of an input layer of $N$ fan-out neurons and an output layer of $K$ processing neurons. The neurons of output layer are full connected to that of input layer. The learning algorithm is shown as follows [4]:

Step 1: Initialize the small random weights.

Step 2: Each output neuron calculates the distance between the input vector and the weight vector connecting the input to it, if the $i^{\text {th }}$ index of the closest competitive neuron is found and set its output activation to 1 , the activation of all other competitive neurons to 0 .

Step 3: The weight of the only winning neuron is updated so as to make the weights of the winning neuron closer to the current input pattern. Thus, the neuron whose weight vector was closest to the input vector is updated to be even closer.

Step 4: Check if the stopping criterion is satisfied, if no more changes of the weight vectors or the maximum epochs is reached, then learning stop, else go to Step 2.

The winning node is more likely to win the competition again when a similar input is presented, and less likely to win when a very different input is presented. Thus, the competitive network clusters the input data, the output vectors of resulting network represent to class indices.

\subsection{RBF Network Classifier}

In the RBF network, the activation function of the hidden nodes is Gaussian function [9], the input of the hidden node $i$ is the product of threshold $b_{i}$ and the Euclidean distance between weight vector $W$ and input vector $X$ : 


$$
\mathrm{k}_{\mathrm{i}}^{\mathrm{q}}=\sqrt{\sum_{j}\left(X_{j}^{q}-W_{j i}\right)^{2}} \times b_{i} .
$$

Where, $X_{j}^{q}$ is the $j^{\text {th }}$ component of the $q^{\text {th }}$ input vector, $W_{j i}$ is the weight between the $j$ th node in the input layer and the $i$ th node in the hidden layer, $b_{i}$ is a threshold to control the accuracy of the Gaussian function. The output of the same node is shown as follows:

$$
\mathrm{r}_{\mathrm{i}}^{\mathrm{q}}=\exp \left(-\left(\mathrm{k}_{\mathrm{i}}^{\mathrm{q}}\right)^{2}\right)=\exp \left(-\sqrt{\sum_{\mathrm{j}}\left(x_{j}^{q}-w_{j i}\right)^{2}} \times \mathrm{b}_{\mathrm{i}}\right) .
$$

Instead of adjusting $b_{i}$, we can use the parameter of spread in Neural Network Toolbox of Matlab 7.0 to control the performance of the network. The larger spread is, the smoother the function approximation will be. The activation function of the network output is linear. The RBF network has a strong capability of approximation to the kernel vector in a limited part of the whole net, its training is divided into two processes. The first is unsupervised learning, which adjusts the weight vector between the input and hidden layers. The other is supervised learning, which adjusts the weight vector between the hidden and output layers.

\section{Experiments}

\subsection{Experimental Data}

The experimental corpus is based on 1204 documents from People's Daily from 1996 to 1998, all of which are first classified manually. These documents are separated into two parts: the training documents and the testing documents. The training corpus contains 1084 documents in six different categories, and the rest 120 documents are used as testing samples, with 20 documents in each category. Instead of using word frequency, we use the combined weighing method CHI-MCOR as features of each document as described in above, the dimension of the feature vector is 500 .

\subsection{Classification by the Competitive Network}

In our experiment, we set the Kohonen learning rate of the competitive network to 0.01 . To prevent the dead nodes problem from happening, we set the conscience mechanism to the competitive network. The network is unable to cluster the documents when conscience rate is larger than 0.001 or too close to zero, thus the conscience rate is set 0.000005 . The clustering result of the competitive network is listed in Table 2.

Table 2. Experimental result of the competitive network

\begin{tabular}{lll}
\hline Positive accuracy & Negative accuracy & Average accuracy \\
\hline 0.5438 & 0.9159 & 0.7299 \\
\hline
\end{tabular}




\subsection{Classification by the RBF Network}

The value of spread is 1.2. After 1084 iterations, the training completes. This process takes only 3 minutes or so. Then another 120 texts are used for testing. Comparing with the classification that has been made manually, we can get the precision, recall and $F 1$ value of the classification made by RBF network. The experimental results are listed in the Table 3.

Table 3. Experimental result of the RBF network, $F 1=0.7722$

\begin{tabular}{llllllll}
\hline & Economics & Politics & Computer & Sports & Education & Law & Average \\
\hline$P$ & 0.615 & 0.435 & 1 & 1 & 0.947 & .722 & 0.7866 \\
$R$ & 0.8 & 0.5 & 0.8 & 0.9 & 0.9 & 0.65 & 0.7583 \\
\hline
\end{tabular}

From the experimental results we can know that the classifier based on competitive network can classify document of different categories correctly, represented by a high negative accuracy rate. However, a low positive accuracy rate shows that many documents from different categories are not clustered together. This is partly because it is difficult to have information about the correct category in the unsupervised learning. In the experiment of the RBF network, the precision and recall are low in some categories, such as Politics category; the performances are fairly nice in Computer and Sports categories. The RBF network performs unsatisfactorily in the same category, the Politics, probably due to that some proper nouns have not been correctly recognized in certain categories and thus lost some important information about the text. Feature words in politics often have vague boundaries with other fields, such as economics, culture, military affaires, and so on. While sports and computer are highly characteristic fields, it is much easier choosing their feature words. Besides, the number of training documents are slightly too small compared to the dimension of the feature vector and the complexity of text classification.

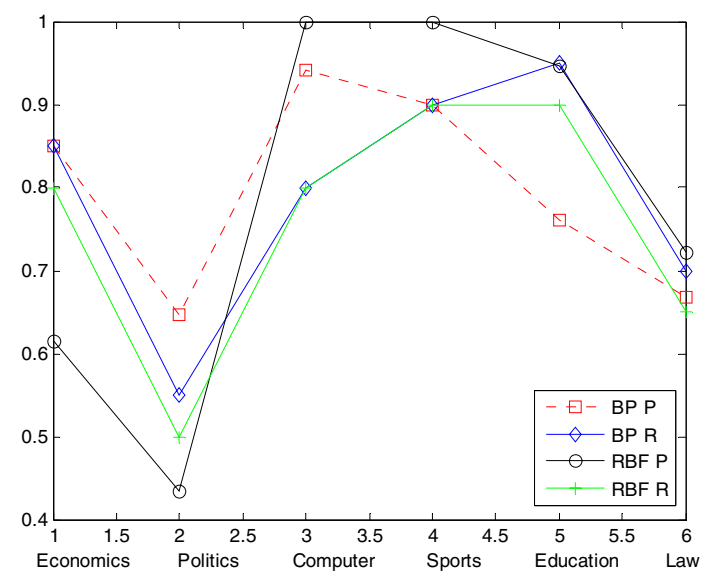

Fig. 2. Performance comparison between the BP and RBF network 
At the same time, we do also experiment of the BP network, the result shows that the RBF network takes less than one-tenth of the training time BP takes, the RBF network performs well in the categories in which the results are satisfactory. In the sports category, it even outperforms the BP network.

\section{Conclusions}

When semantic sememes are used as the features set of text classification, we can efficiently reduce the feature dimension and reflect the original feature space to a more stable and smaller one. By setting a proper threshold, we can reserve the word whose concept definition is weak in expression. Meanwhile, as every sememe has its own expression power, we give them different values according to their expression power and relation to the word. Experimental results show that this combined feature set is much better than the word one or the semantic one. Because there are some words which are not highly occurred but useful in text classification, while the words with high occurrence frequency is usually useful, except the words in the stop word dictionary which are frequently used in the text but useless in classification, our CHIMCOR method to take balance in the high occurring ones and the middle occurring ones. This method not only selects the highly occurring words, but also selects the words whose occurrence frequencies are middle or low but only occur in one or two categories. It is much better than $\mathrm{CHI}$ or MCOR method alone.

The competitive network sometime cannot cluster documents of the same category together, because it lacks category information as a method of unsupervised learning. As of the supervised learning methods, the RBF network shows its quickness in training and it can even outperform the BP's capability, especially being modified in some means. As a classifier, the RBF network can be a good substitute for the BP network, when the selected features are clear enough for the RBF network itself to produce satisfactory results.

\section{Acknowledgement}

This work was supported by National Natural Science Key Foundation of China (No. 60331010) and National Natural Science Foundation of China; Tsinghua University 985 Research Foundation; the Excellent Young Teacher Program, Ministry of Education, China; and State Key Lab of Pattern Recognition Open Foundation, Chinese Academy of Sciences.

\section{References}

1. Liao, S., Jiang, M.: An Improved Method of Feature Selection Based on Concept Attributes in Text Classification. In: Wang, L., Chen, K., and Ong, Y. S. (eds.): ICNC 2005, Lecture Notes in Computer Science, 3610 (2005) 1140 - 1149

2. Mlademnic, D., Gtobelnik, M.: Feature Selection for Unbalanced Class Distribution and Naïve Bayees. Proceedings of the Sixteenth International Conference on Machine learning, (1999) 258-267 
3. Yang, Y., Pedersen, J. O.: A Comparative Study on Feature Selection in Text Categorization. In: Proceedings of the 14th International Conference on Machine Learning, (1997) 412-420

4. Wang, L., Jiang, M., Lu, Y., Noe, F., and Smith, J. C.: Clustering Analysis of Competitive Learning Network for Molecular Data. In: Wang, J. et al (eds.): ISNN06, Lecture Notes in Computer Science, 3971 (2006) 1244-1249

5. Wang, L., Jiang, M., Lu, Y., Noe, F., and Smith, J. C.: Self-Organizing Map Clustering Analysis for Molecular Data. In: Wang, J. et al (eds.): ISNN06, Lecture Notes in Computer Science, 3971 (2006) 1250-1255

6. Dong, Z., Dong, Q.: The Download of Hownet [EB/OL], http://www.keenage.com

7. Wang, L., Jiang, M., and Liao, S., et al.: A Feature Selection Method Based on Concept Extraction and SOM Text Clustering Analysis. International Journal of Computer Science and Network Security, 6 (1A), (2006) 20-28

8. Liao, S., Jiang, M.: A Combined Weight Method Based on Concept Extraction in Automatic Classification of Chinese Text. Proceedings of 2005 IEEE International Conference on Neural Networks and Brain, (2005) 625-630

9. Martin, T., Hagan, H., Demuth, B., and Beale, M.: Neural Network Design. PWS Publishing Company (1996) 